\title{
Update on the clinical management of Wilson's disease
}

\section{Peter Hedera}

Department of Neurology, Vanderbilt University Medical Center, Nashville, TN, USA
Correspondence: Peter Hedera Department of Neurology, Vanderbilt University Medical Center, 465 2I st Avenue South, 6140 MRB III, Nashville, TN 37232, USA

Tel + I 6159363920

Fax +l 6I5 3220486

Email peter.hedera@vanderbilt.edu
This article was published in the following Dove Press journal:

The Application of Clinical Genetics

13 January 2017

Number of times this article has been viewed

\begin{abstract}
Wilson's disease (WD), albeit relatively rare, is an important genetic metabolic disease because of highly effective therapies that can be lifesaving. It is a great imitator and requires a high index of suspicion for correct and timely diagnosis. Neurologic, psychiatric and hepatologic problems in WD are very nonspecific, and we discuss the most common clinical phenotypes. The diagnosis remains laboratory based, and here we review the most important challenges and pitfalls in laboratory evaluation of WD, including the emerging role of genetic testing in WD diagnosis. WD is a monogenic disorder but has very high allelic heterogeneity with $>500$ disease-causing mutations identified, and new insights into phenotype-genotype correlations are also reviewed. The gold standard of therapy is chelation of excessive copper, but many unmet needs exist because of possible clinical deterioration in treated patients and potential adverse effects associated with currently available chelating medications. We also review the most promising novel therapeutic approaches, including chelators targeting specific cell types, cell transplantation and gene therapy.
\end{abstract}

Keywords: Wilson's disease, copper, ATP7B, chelation, gene therapy

\section{Introduction}

Wilson's disease (WD) is an inherited disease of copper transport caused by loss of function of the $A T P 7 B$ copper-binding protein. ${ }^{1,2}$ The resulting defect in hepatic excretory pathway with impaired excretion of copper into the bile leads to accumulation of copper with cytotoxic changes in the liver and other tissues, most notably the central nervous system. WD may present as hepatic, neurologic or psychiatric problems. ${ }^{3}$ The diagnosis remains laboratory based, but the availability of genetic testing may further increase clinical certainty, especially in heterozygous patients or patients with biliary obstruction. We also review the emerging phenotype-genotype correlation. The gold standard of therapy is chelation of excessive copper, but many unmet needs exist because of possible clinical deterioration in treated patients. This review focuses on novel therapeutic approaches, including gene therapy for WD.

\section{Clinical presentation}

Clinical features associated with symptomatic WD are protean, and it is an ultimate great imitator requiring a high index of suspicion for timely diagnosis. ${ }^{3}$ The age of first symptoms varies widely from the first decade to the fourth and fifth decades of life, even though most patients develop symptoms in adolescence to early adulthood. ${ }^{4-6}$ Neurologic symptoms tend to develop later by nearly one decade than hepatic presentation, but 
there is a considerable overlap between these two major clinical phenotypes. Atypical, late onset of disease may represent a significant diagnostic challenge because clinical symptoms are similar to other common, age-related conditions. ${ }^{7}$

Initial signs and symptoms of WD are hepatic in $\sim 40 \%$ of patients, neurologic in $\sim 40 \%-50 \%$ and primarily psychiatric manifestation can be seen in $\sim 10 \%$ of patients. ${ }^{3,4,8}$ However, most of them typically develop combined hepatic and neuropsychiatric symptoms. Approximately $10 \%$ are asymptomatic when diagnosed with WD, and they are typically diagnosed during the screening of siblings of affected probands or during routine laboratory test when unexplained elevation of liver enzymes is detected.

Hepatic involvement may range from an asymptomatic state to life-threatening hepatic failure, and in $\sim 5 \%$ of patients it rapidly evolves as a fulminant WD from seemingly normal state of health. ${ }^{89}$ Asymptomatic patients typically have only biochemical abnormalities with elevation of liver enzymes and histologic findings of steatosis on liver biopsy. Liver involvement in WD may also mimic acute viral hepatitis or autoimmune hepatitis. However, most patients with hepatic symptoms exhibit signs of chronic liver disease with cirrhosis and splenomegaly due to portal hypertension. Hepatic phenotype of WD is frequently associated with Coombs-negative hemolytic anemia that can lead to an acute renal failure in extreme cases. ${ }^{10}$ Transient episodes of jaundice due to hemolysis may be the initial presentation in patients who do not have any other signs of liver disease.

Early neurologic symptoms may be very subtle and nonspecific with subjective difficulties in concentration, lack of coordination, changes in penmanship and slurred speech with drooling. ${ }^{11,12}$ They may be intermittent, and in early stages the neurologic examination can be unremarkable. Somatization or psychogenic condition is often misdiagnosed with potentially catastrophic consequences because of delayed chelation therapy. More pronounced neurologic abnormalities encompass dysarthria, dystonia, tremor and parkinsonian manifestations. However, they typically overlap in more advanced stages, and dysarthria is present in the majority of all patients. Many patients with neurologic symptoms do not have any obvious hepatic symptoms.

Tremor is one of the most common neurologic signs present in $22 \%-55 \%$ of patients diagnosed with WD. ${ }^{13,14}$ Prototypical tremor in WD is described as the wing-beating tremor with proximal tremor, appearing when the patient holds semiflexed outstretched arms. Characteristically, its amplitude increases with a longer duration of posture holding, and many patients exhibit severe flapping tremor with large amplitudes. However, many patients suffer from more typical postural and action tremor that can be easily confused with essential tremor, which is a very common neurologic entity affecting $0.5 \%-5 \%$ of the general population. ${ }^{15}$

Dystonia is another common neurologic manifestation of WD and present in $\sim 10 \%-65 \%$ of all patients. ${ }^{12-14}$ It is characterized by an abnormal posture of various body segments commonly associated with twitching or twisting movements. It can be focal, such as forceful eye closure due to blepharospasm or segmental, such as involuntary head rotation associated with unilateral shoulder elevation as seen in spasmodic torticollis. Segmental or focal dystonia in craniofacial region is especially very symptomatic with severe dysphonia, dysarthria, risus sardonicus with forced, often exaggerated smile and dysphagia with a complete loss of speech and inability to swallow. Advanced WD may also lead to generalized dystonia, resulting in abnormal posturing of the trunk, neck or extremities and subsequently causing debilitating symptoms with secondary skeletal changes and inability to walk.

Slowness of movements with rigidity and bradykinesia may superficially resemble Parkinson's disease, but WD has rarely an asymmetric onset. Masked face with hypophonic, soft voice, micrographia and shuffling or freezing gait are most frequent and typical parkinsonian features. Less common neurologic problems include spasticity, chorea, athetosis or myoclonus.

The third most common type of presenting symptoms are psychiatric and behavioral symptoms. ${ }^{16,17}$ They are also very nonspecific and can range from depression to acute psychotic episodes. Frank psychosis is present in $\sim 10 \%$ of these patients and is frequently misdiagnosed as schizophrenia or bipolar disease. Behavioral and personality changes commonly include attention deficit, compulsive or impulsive behavior and apathy. Overall, approximately half of all patients with manifesting WD have active psychiatric problems. They are usually accompanied by additional neurologic signs, but these initial neurologic signs can be very subtle and easily overlooked or attributed to the use of neuroleptics that were used to treat the psychiatric condition. However, clearly some patients, estimated between $10 \%$ and $20 \%$, may present with truly isolated psychiatric symptoms without any neurologic or hepatic manifestations. ${ }^{17}$ Analysis of patients who were initially seen by a psychiatrist showed that the average delay of diagnosis was $>2$ years because isolated psychiatric symptoms without obvious neurologic or hepatic involvement do not prompt laboratory evaluation for possible WD. ${ }^{16-18}$

WD as an autosomal recessive disorder affects men and women equally, but there are important sex-specific 
phenotypic differences. Hepatic forms are seen more frequently in women who develop it in $55 \%-60 \%$ of all cases. Women also tend to have a later onset of symptoms for $\sim 2$ years later than men. ${ }^{4,19}$ No obvious differences in the degree of copper overload in women have been found, and the influence of estrogens has been suggested as one possible explanation of this sex difference. ${ }^{19}$

Other systemic manifestations of WD are rare and very difficult to recognize as WD without telltale signs of hepatolenticular degeneration. Aminoaciduria, nephrolithiasis, arthropathy, premature osteoporosis and cardiomyopathy have been reported as unusual presenting symptoms of WD. ${ }^{20-23}$ Ophthalmologic features are also common, and they are typically asymptomatic but may be useful in supporting the diagnosis. The most important are Kayser-Fleischer rings that are caused by asymptomatic copper deposition in Descemet's corneal membrane. They may be visible by naked eye as a golden-brownish pigmentation around the limbus. Some patients may not have a fully formed circle and increased pigmentation can be seen at $\sim 6$ and 12 o'clock position. Definitive detection of Kayser-Fleischer rings should be established using slit lamp examination. This examination can also detect asymptomatic sunflower cataracts that are caused by copper deposits in the lens. The presence of rings can only support the diagnosis because rarely they can be seen in patients with chronic cholestasis. ${ }^{24}$ Additionally, they may be absent in some fully symptomatic patients, and almost half of patients with an isolated liver disease does not have fully formed rings. ${ }^{4,25}$ Their absence is rare in patients with neurologic presentation and only $\sim 10 \%$ of these patients have a normal slit lamp examination. ${ }^{26}$ Copper deposits in the cornea and the lens disappear with chelation, but there is no correlation between their presence and the severity of clinical deficits.

\section{Diagnosis of WD}

Broad age of onset with variable and nonspecific clinical presentations requires a high index of suspicion for early diagnosis. Delayed recognition of the disease can lead to a progressive worsening of hepatic and neurologic symptoms, and this may influence the overall prognosis for improvement. ${ }^{27,28}$ Treatment outcomes are best if the interval between first symptoms and initiation of therapy is $<1$ month. ${ }^{18}$ When delay in the diagnosis was increased to $1-6$ months, only about one-fifth of patients achieved a favorable outcome with very minor disability. However, average time from the symptom onset to a correct diagnosis and appropriate treatment is still unsatisfactory with an average of $\sim 1$ year between the onset and diagnosis. This is one of the reasons that many patients experience disabling neurologic problems or irreversible structural hepatic changes. ${ }^{18}$

The diagnosis of WD remains laboratory based and suggestive clinical phenotypes, as outlined above, should prompt further laboratory testing. Two sets of diagnostic algorithms have been published and are widely accepted, including the scoring system for the diagnosis. ${ }^{8,29}$ However, they are more pertinent for patients with hepatic presentations because it may be more difficult to differentiate WD from other hepatologic conditions. Patients presenting with neurologic or psychiatric symptoms tend to have more straightforward laboratory diagnosis because the cause of abnormal copper homeostasis is less obscure. We discuss existing pitfalls in laboratory evaluation based on published recommendations.

Plasma levels of ceruloplasmin are recommended as the first step in the diagnosis of WD. ${ }^{8}$ It remains a screening laboratory assay and even low levels cannot confirm the diagnosis, and additional confirmatory tests are needed. A serum ceruloplasmin level $<20 \mathrm{mg} / \mathrm{dL}(200 \mathrm{mg} / \mathrm{L}$ or $2.83 \mu \mathrm{mol} / \mathrm{L}$ ) is consistent with the diagnosis but overall the positive predictive value is very low at $5.9 \% .{ }^{30}$ Ceruloplasmin is the main copper-binding plasma protein with $>90 \%$ of total copper bound to it. It exists as holoceruloplasmin with bound copper and apoceruloplasmin that does not contain copper. ${ }^{31}$ Almost exclusively it is measured by antibody-based radioimmunological assays that cannot differentiate between these two isoforms of ceruloplasmin, causing an overestimation of copper-binding protein in plasma. Levels of serum ceruloplasmin may also be measured enzymatically by their copper-dependent oxidase activity. ${ }^{32}$ This assay can distinguish between apoceruloplasmin and holoceruloplasmin, but it is not widely available.

Ceruloplasmin is also an acute-phase reactant, and this is another common cause of higher levels that may lead to missed diagnosis of WD. Additional important cause of higher ceruloplasmin is elevated estrogen levels, most commonly induced by birth control pills or estrogen replacement therapy. ${ }^{33}$ Abnormally low ceruloplasmin levels $<5 \mathrm{mg} / \mathrm{dL}$ are strongly suggestive of WD, but such low values can also be found in conditions with very low copper plasma values, especially with copper deficiency and aceruloplasminemia, which is a rare cause and an autosomal recessive condition caused by mutations in the ceruloplasmin gene. ${ }^{34,35}$ Neurologic clinical presentation of aceruloplasminemia may mimic WD but the pathogenesis is actually iron overload. Another possible cause of low ceruloplasmin is Menkes disease, an $\mathrm{X}$-linked disorder of copper transport from enterocytes to 
blood and through the blood-brain barrier (BBB) caused by mutations in the $A T P 7 A$ gene. ${ }^{36,37}$ Heterozygotes carrying one mutated allele of $A T P 7 B$ gene may also have borderline low values requiring further testing. ${ }^{8}$

Every patient with suspected WD needs to have 24-hour urine copper assay, and this test alone can be diagnostic in many patients. It is important to completely collect 24 -hour urine starting after the first morning voiding in the day of the collection day and complete it the next day after the first voiding. Another technical requirement is a copper-free collection vessel. Total creatinine excretion in the 24-hour urine collection is typically measured to support proper urine collection. The 24-hour copper values $>100 \mu \mathrm{g} / 24 \mathrm{~h}$ $(1.6 \mu \mathrm{mol} / 24 \mathrm{~h})$ is conventionally considered diagnostic of WD. ${ }^{8}$ This is definitely true for patients with neurologic or psychiatric phenotypic presentations. However, patients with chronic cholestasis or autoimmune hepatitis may also have values $>100 \mu \mathrm{g} / 24 \mathrm{~h}$, and additional testing is necessary to distinguish this from WD. ${ }^{8}$

Normal values for 24-hour excretion somewhat vary for different laboratories but typically are $<40$ or $50 \mu \mathrm{g}$ ( 0.64 or $0.8 \mu \mathrm{mol}) / 24 \mathrm{~h}$. The 24 -hour urine copper values below this cutoff exclude WD. Intermediate values between 40/50 and $100 \mu \mathrm{g} / 24 \mathrm{~h}$ may be seen in heterozygous (carrier) individuals and require further investigation. Affected symptomatic children with WD may also have 24-hour urine copper values below the conventional cutoff and lowering this value in pediatric patients has been suggested. ${ }^{38-40}$ Twenty-four-hour urine copper test can also be used for therapy monitoring.

D-Penicillamine challenge has been used in patients with borderline 24-hour urine copper values but this test has been validated only in children with hepatic presentation. Administration of $500 \mathrm{mg}$ of D-penicillamine before urine collection and repeated once 12 hours into the collection promotes cupriuria and the values of more than $1,600 \mu \mathrm{g}$ copper/24 h $(25 \mu \mathrm{mol} / 24 \mathrm{~h})$ are considered diagnostic. This test is not suitable for identification asymptomatic siblings at risk because it can identify only about half unaffected WD patients. Overall, this test is used less commonly with the availability of genetic testing for $A T P 7 B$ mutations. ${ }^{41,42}$

Assay of liver copper content has been considered a gold standard for the confirmation of the diagnosis and may be still required in patients with predominantly hepatic presentation. However, the diagnosis of neurologic or psychiatric WD is sufficient based on diagnostic values of 24-hour urine copper excretion. Hepatic copper content $>250 \mu \mathrm{g} / \mathrm{g}$ dry weight $(4.0 \mu \mathrm{mol} / \mathrm{g}$ of tissue) is considered diagnostic for WD. ${ }^{8}$ Normal hepatic copper concentration rarely exceeds
$50 \mu \mathrm{g} / \mathrm{g}(0.8 \mu \mathrm{mol} / \mathrm{g})$ of tissue dry weight. However, lowering of this cutoff value may reduce the sensitivity because long-standing cholestatic disorders and heterozygotes have also commonly elevated liver copper content but very rarely $>250 \mu \mathrm{g} / \mathrm{g}(4.0 \mu \mathrm{mol} / \mathrm{g})$ dry weight. $^{8}$

Additional potential laboratory WD marker is assaying plasma copper. Total serum copper consists of bound and free (unbound) copper. Total copper value alone is not very helpful in the diagnosis of WD because it is very variable and typically parallels the main copper-binding protein ceruloplasmin. Most laboratories consider $65-140 \mu \mathrm{g} / \mathrm{dL}(10-22 \mu \mathrm{mol} / \mathrm{L})$ range as normal. ${ }^{8}$ However, a significant attention has been devoted to ceruloplasmin unbound or free plasma copper that better reflects copper homeostasis because only free copper is biologically active. Under normal conditions, $5 \%-15 \%$ of total copper is unbound to ceruloplasmin. It can also be mobilized with chelation therapies and spikes in free plasma copper are thought to be responsible for neurologic progression after the therapy was initiated. ${ }^{3}$ That is why free copper values are also even more important for the monitoring of therapy.

Free copper can be calculated from assayed total plasma copper and ceruloplasmin. Six copper atoms are bound to one molecule of ceruloplasmin, resulting in $\sim 3.15 \mu \mathrm{g}$ of copper weight $/ 1 \mathrm{mg}$ of ceruloplasmin. Thus, free copper can be estimated as a difference between the total copper and ceruloplasmin value multiplied by three. ${ }^{3,8}$ However, this needs to be interpreted with caution, especially when the levels of ceruloplasmin are low. Free copper can be directly measured but this is limited to a few commercial laboratories.

Serum nonceruloplasmin-bound copper concentration has been proposed as a diagnostic test for WD. It is elevated $>25 \mu \mathrm{g} / \mathrm{dL}(250 \mu \mathrm{g} / \mathrm{L})$ in most untreated patients but may also be seen in acute liver pathology, diminishing its specificity. Normal values are $10-15 \mu \mathrm{g} / \mathrm{dL}(100-150 \mu \mathrm{g} / \mathrm{L}$ or $1.6-2.4 \mu \mathrm{mol} / \mathrm{L})$, and free copper $<5 \mu \mathrm{g} / \mathrm{dL}(0.84 \mu \mathrm{mol} / \mathrm{L})$ indicates copper deficiency that may also occur iatrogenically in treated WD patients. ${ }^{43}$ Limited availability of direct measurement of free copper reduces the value of free copper in the diagnosis of WD, but novel therapeutic approaches focus on tighter control of unbound copper and this is further discussed in the section of Treatment of WD.

A scoring system utilizing these clinical and laboratory features, including the presence of Kayser-Fleischer rings, neurologic or neuroimaging features, hemolytic anemia, elevated liver function test, elevated 24-hour urine copper values, reduced ceruloplasmin and mutation analysis, has been developed. ${ }^{29}$ The total score is generated by adding values from zero (normal examination or absent laboratory 
abnormalities) and two points (abnormal clinical signs or abnormal laboratory tests present) to four points if both mutations are detected. Overall score of more than four points is highly suggestive for WD, and the score between two and three points is classified as probable WD where more diagnostic tests are needed. Application of this scoring system in a cohort of 142 children with liver disease only yielded a sensitivity of $98.14 \%$, a specificity of $96.59 \%$, a positive predictive value of $94.64 \%$ and a negative predictive value of $98.83 \%{ }^{44}$

Structural and to some extent functional neuroimaging may be helpful in the diagnosis of WD. ${ }^{45-47}$ Magnetic resonance imaging (MRI) detects brain abnormalities in essentially every patient exhibiting neurologic symptoms. However, these changes are nonspecific, and WD needs to be confirmed by other laboratory methods. Hyperintensity on T2 weighted and fluid-attenuated inversion recovery images are characteristically present in putamen that is the most commonly involved basal ganglia structure, followed by striatum and globus pallidus. ${ }^{45,46} \mathrm{~A}$ thin rim of T2 hyperintensity in the claustrum is also frequently seen and it is known as the bright claustrum sign. These abnormal signal changes may spread to brain stem. Hyperintense signal in the midbrain around the red nucleus and substantia nigra may give the appearance of "panda sign" that is most commonly seen in WD patients. Widespread white matter changes resembling leukodystrophy can be rarely seen in the centrum semiovale and are typically associated with poor prognosis and severe deficits. ${ }^{48}$ Structural brain changes result from copper toxicity but the underlying hepatic encephalopathy also has MRI correlates in T1-weighted hyperintensity affecting the same areas of brain as seen on T2-weighted images. Patients with liver failure may have only T1-weighted abnormalities on their brain MRI scans without typical T2 changes in basal ganglia. ${ }^{45,49}$ Advanced WD with persisting neurologic morbidity cause secondary brain atrophy but this is seen in later stages of the disease.

MRI changes can be evaluated as qualitative assessment but also a scoring system of imaging abnormalities has been proposed using the degree of signal changes and degree of cortical atrophy. ${ }^{50}$ MRI changes correlated with neurologic deficits and clinical improvement with chelation therapy also followed improvement of serial MRIs. Structural changes also correlate with cognitive decline in these patients who typically exhibit frontal lobe dysfunction. ${ }^{51}$ Other imaging modalities are also used in evaluation of WD patients. Magnetic resonance spectroscopy demonstrated a reduction of $\mathrm{N}$-acetylaspartate and $\mathrm{N}$-acetylaspartylglutamate in patients with neurologic features but not in patients with hepatic involvement only. ${ }^{52}$ Functional assessment of dopaminergic innervation using single-photon emission computerized tomography (SPECT) in striatum can detect abnormalities in both presynaptic and postsynaptic dopaminergic markers. This pattern is relatively specific for WD because neurodegenerative striatonigral degeneration usually shows either pre- or postsynaptic deficits only. ${ }^{53}$ Glucose uptake in basal ganglia is also reduced in WD, but this is a very nonspecific finding and does not differentiate between WD and other neurodegenerative conditions. ${ }^{31}$ Moreover, there is no established correlation between functional defects and clinical picture using either cross-sectional or longitudinal studies.

Ultrasound, computed tomography (CT) and MRI of the liver in WD usually reflect nonspecific hepatic injury, hepatitis and cirrhosis. ${ }^{54,55}$ Nodular infiltrations suggesting the presence of copper-containing nodules and contour abnormalities consistent with cirrhosis may be seen in advanced WD. Follow-up liver imaging can show reduction of copper content in the liver, paralleling clinical improvement. ${ }^{55}$ Noninvasive methods for liver fibrosis assessment include elastographic methods involving ultrasonography and MRI. This is a promising noninvasive method allowing monitoring fibrotic changes in response to chelation therapy in WD. ${ }^{56}$

Patients where diagnosis of WD cannot be unequivocally confirmed using these approaches may be suitable for genetic testing. We review the role of mutation analysis in the following section.

\section{Genetics of WD}

WD is an autosomal recessive disorder caused by mutations in the $A T P 7 B$ gene resulting in deficient production of the copper-transporter adenosine triphosphatase 2 (ATPase2). ${ }^{1,2}$ This protein has a double role in copper homeostasis. ${ }^{57}$ Its housekeeping function is transferring copper to the Golgi complex to make it available to the proteins requiring copper as a cofactor. Its function modifies when excessive intracellular copper levels are present. ATP7B changes its intracellular localization to the apical membrane of hepatocytes to promote copper excretion into the bile. ${ }^{57}$ Thus, its loss of function leads to abnormal copper distribution and copper overload.

There is no evidence for genetic heterogeneity and mutations in this gene account for every case of WD. Overall prevalence is estimated between 1:30,000 and 1:40,000 with the corresponding carrier frequency $1: 90$ to $1: 100 .^{58-60}$ Several populations with much higher prevalence have been identified, and this is mainly from founder effects, such as in Sardinia where the disease frequency is estimated from $1: 10,000$ to $1: 7,000$. The founder's mutation with 
15-nucleotide deletion in the promoter region (-441/-427del) accounts for $61.7 \%$ of all WD alleles in Sardinia ${ }^{61}$ Another region with a higher than background prevalence is Costa Rica with 1:20,400 (4.9:100,000) prevalence. ${ }^{62}$

Estimates of gene frequencies are based on already diagnosed patients with WD. Recent genetic investigation of 181 patients from the UK with confirmed WD suggested that the frequency of heterozygosity is considerably higher than the previously reported occurrence of $1: 100 .^{63}$ The calculated frequency of individuals predicted to carry two pathogenic mutations using Hardy-Weinberg law was 1:7,026 that is considerably higher than the reported prevalence of WD of $1: 30,000$ or $1: 40,000$. It remains unclear whether this discrepancy between the genetic prevalence and the number of clinically diagnosed cases of WD is due to an unrecognized disease or by the fact that some mutations may have reduced penetrance of the disease. WD was confirmed in two siblings in their 70s, confirming that mild and atypical forms of WD exist and are rarely diagnosed. ${ }^{7}$

More than 500 disease-causing mutations, in addition to $\sim 100$ nonpathogenic variants, have been identified in the $A T P 7 B$ gene. This means that many mutations are private, limited to single families, and the vast majority of patients are compound heterozygotes. Unless familial mutations are known or the patient is from the region where certain mutations are very common, all exons and promoter region need to be sequenced. Targeted mutation analysis for specific mutations, such as multiplex amplification refractory mutation system polymerase chain reaction, may be helpful in populations with prevalent common mutations, including patients of the southeastern Asian descent who harbor R778L mutation in $58 \%$ of all WD cases. ${ }^{64}$ Constant progress in sequencing methods and improved bioinformatics methods increases the success in mutation detection, and the highest reported detection rate was $98 \%$ in a cohort with laboratoryproven WD. ${ }^{63}$ Other studies reported lower success with the detection rate of $68.5 \%$, and in $7 \%$ of patients no mutation was detected on either allele. ${ }^{65}$ Genetics testing can confirm WD only when biallelic pathogenic mutations are identified. Gene dosage assays can be helpful to detect deletions that cannot be identified by sequencing methods. Thus, at present, negative genetic testing alone does not fully exclude WD and other laboratory methods need to be considered.

In spite of allelic heterogeneity in the $A T P 7 B$ gene, several groups attempted to analyze phenotype-genotype correlation data. Truncating mutations are associated with fulminant hepatic failure and an early age of onset, suggesting that a complete loss of function leads to early copper overload. ${ }^{66,67}$
Contrary to this, patients carrying H714Q mutation tend to have late, neurologic presentation. ${ }^{68}$ This mutation, found in $\sim 20 \%$ of patients, affects the phosphorylation domain and has some residual functional activity, likely accounting for a slower accumulation of copper and hence, later onset of the disease. The most common mutation among the Caucasian patients from Europe and North America is H1069Q and is located in the loop motif with the disruption of ATP binding. This mutation accounts for $\sim 40 \%$ of all mutations, especially in individuals from Eastern Europe and Germany. ${ }^{69}$ Homozygosity for this mutation is relatively common to allow a meaningful phenotypic-genotypic correlation. However, homozygous patients have almost equal chance for either neurologic (51.7\%) or hepatic (48.3\%) presentations. Overall, the presence of this mutated allele was seen in $39.8 \%$ patients with hepatic and $51.9 \%$ with neurologic presentations. ${ }^{65}$

Consanguineous marriages increase the likelihood of homozygous mutations, and genetic analysis of these patients is an important tool in genotype-phenotype analysis. Reported consanguinity in WD in regions with frequent inbreeding varies from $30 \%$ to $90 \%{ }^{62,70-72}$ Even though the detected mutations in these populations represent only a fraction of all known mutations, several conclusions can be drawn. Homozygosity of N1270S predisposes to liver disease with frequent fulminant liver failure as presenting feature, c.2299insC predisposes to hepatic and A1003T to neurologic symptoms. ${ }^{62,71}$

This marginal difference is not clinically useful and further supports important roles for genetic modifiers and perhaps environmental factors. Comparisons of phenotypes among siblings of second-degree relatives showed relatively high intrafamilial concordance of the WD phenotype ${ }^{73}$ This suggests that siblings tend to have similar clinical problems and this type of information may be more useful for management of other family members.

Genetic testing of WD can be helpful in certain circumstances, but the described challenges and pitfalls prevent its implementation as a routine and first-line diagnostic test. Patients whose WD is suspected but cannot be unequivocally confirmed by laboratory evaluation may benefit from genetic testing, but only the detection of both mutated alleles can confirm the diagnosis. Negative testing may reduce the likelihood of WD, but clearly there are mutations that are not detected in patients with confirmed WD. Genetic testing can also improve the identification of unaffected siblings who are at risk. Children aged $<10$ years may have nondiagnostic values of 24-hour urine copper or liver copper content, and genetic analysis can confirm or exclude the diagnosis. ${ }^{38,39}$ 
However, this is only possible if both familial mutations have been definitely identified. Genotypic data are only marginally helpful to predict the type of clinical presentation, and its usefulness is limited to affected siblings who tend to have a degree of phenotypic concordance. However, in large cohorts of unrelated patients, genotype does not predict phenotype of WD.

\section{Treatment of WD}

Untreated WD inevitably leads to hepatic, neurologic or psychiatric problems, or their various combinations. The treatment goals in WD are to reverse copper overload and establish a negative copper balance during which copper values are reduced to normal levels. The second step is maintaining these levels without inducing iatrogenic copper deficiency. Maintenance therapy starts after disease symptoms and biochemical abnormalities have improved, and this is typically observed in 2-6 months after initiation of chelation therapy. Unless the patient undergoes liver transplant, the maintenance phase represents a lifelong therapy. The current treatments for WD are chelator therapies with D-penicillamine and trientine (also known as triethylenetetramine [TETA]), which nonspecifically chelate copper and promote urinary copper excretion. They can also be used for maintenance therapy. Additionally, zinc is used to induce negative copper balance but its mechanism of action is different from chelators. It is a potent inducer of metallothioneins, which block dietary uptake of copper in enterocytes. Given the delay of several months in its peak efficacy, zinc is used mainly for maintenance treatment. ${ }^{3,8}$

Zinc is also a safe and effective option during pregnancy. ${ }^{74}$ It is the only available medication that does not have to be adjusted in pregnant women. Poorly controlled WD usually results in infertility or early miscarriages. Thus, women who got pregnant have generally good copper control, and it is important to maintain copper balance during the whole pregnancy to prevent acute liver failure or emergence of new neurologic deficits. Successful pregnancies have been reported on trientine and D-penicillamine. ${ }^{75-77}$ Dose reduction of chelating medications is recommended in the range of $25 \%-50 \%$ with urine copper monitoring on a monthly basis until the delivery. ${ }^{8}$

Some patients experience further worsening, especially of neurologic symptoms, even after an appropriate therapy is initiated in a timely manner. The presumed mechanism behind this worsening is a rapid mobilization of copper from the liver leading to elevations in blood-unbound copper, resulting in neurologic deterioration in treated patients. This effect is more common in patients treated with D-penicillamine and up to $50 \%$ of WD patients with neurologic presentation experience further deterioration that is often irreversible. ${ }^{78}$ The same phenomenon is also observed with trientine therapy but its incidence is lower with $10 \%-15 \%$ patients experiencing progression of their neurologic symptoms. ${ }^{79}$ Additionally, D-penicillamine is also associated with many side effects, including fever, lupus-like reaction, bone marrow suppression, nephrotic syndrome, elastosis perforans serpingosa that is a type of skin degeneration or hepatotoxicity, and many patients poorly tolerate this medication. ${ }^{3}$ Trientine is better tolerated, and nephrotic syndrome and aplastic anemia may rarely occur. ${ }^{3}$ This leaves us with many unmet needs for effective and safe WD therapies. Treatment algorithms for WD patients have been extensively reviewed, and we will not further discuss currently available medications to treat WD. ${ }^{8,29}$ The focus of this section is on novel therapeutic agents and novel therapeutic approaches, such as gene therapy.

Ammonium tetrathiomolybdate (TM) is not approved by the US Food and Drug Administration, but it is currently available on a limited basis in Europe since 2008 to treat WD. However, bis-choline TM (WTX101) is now actively studied in WD (ClinicalTrials.gov Identifier: NCT02273596; a phase II, multicenter, open-label, study to evaluate the efficacy and safety of WTX101 administered for 24 weeks in newly diagnosed WD patients aged $\geq 18$ years with an extension phase of 36 months). TM has also been extensively studied in the recent past, and this compound provides several potential advantages and is one of the most promising new agents, especially for patients with neurologic presentation. ${ }^{80-84}$

TM has a double mechanism of action based on its administration with or without food. It contains four sulfur groups that can form a tripartite, very stable complex with copper and albumin (copper-TM protein) ${ }^{80}$ Its administration between meals results in a high degree of absorption and it forms complexes in plasma with present free copper. These insoluble copper complexes are then deposited in the liver, rendering copper biologically inactive. Its administration with meals results in the same complexes with copper contained in food, and this copper cannot be absorbed.

TM has been mostly studied in patients with neurologic WD phenotype. ${ }^{79-84}$ It improved neurologic symptoms without neurologic worsening in 53 from 55 studied patients using an open-label design. ${ }^{83}$ The follow-up study was designed as a double-blind comparison of TM and trientine. One-quarter of patients treated with trientine had neurologic worsening $(6 / 23)$, while this problem was observed only in $4 \%$ patients 
on TM (1/25).$^{79}$ Potential adverse effects included bone marrow depression and hepatotoxicity.

Most likely explanation of the superior efficacy and safety of TM is its control of free copper levels. ${ }^{84} \mathrm{TM}$ exhibited a good control of free copper levels over the 8 weeks of treatment in the 55-patient open-label study, reducing it to a mean value of about one-fourth, or less, of baseline. In the TM/trientine double-blinded study, TM also showed good control of free copper levels, while in the trientine arm, mean free copper levels actually increased during acute chelation therapy. Furthermore, the patients with observed neurologic deterioration had significant spikes in serum-free copper levels associated in time with their neurologic worsening. Patients on trientine who did not progress did not have similar fluctuations of free copper levels. ${ }^{84}$ Thus, further advantage of TM is a tight control of free copper levels that is not easily achievable with either trientine or D-penicillamine.

Additional novel approaches are organ-specific chelators that would exhibit its copper-binding function without mobilization of available copper pool. The main organ of copper regulation is the liver, and the concept of specific copper chelators acting intracellularly in hepatocytes has been proposed. This strategy for chelating intracellular copper is expected to avoid systemic toxicity caused by excessive release of copper from the liver and thus, maintaining free copper plasma in the safe range. One example of this concept is a novel chelator that is a tripodal cysteine-based ligand inspired by metallothioneins. ${ }^{85,86}$ Its high affinity to the liver has been achieved with specific recognition elements of the asialoglycoprotein receptor that is specific for targeting hepatocytes. Further preclinical studies are needed, but this concept may be feasible to avoid toxic effects of systemic chelators.

All available chelators do not cross the BBB, and copper removal from the central nervous system is achieved by its mobilization and shift to plasma pool where it can be chelated and removed.$^{87}$ One strategy to overcome this obstacle is the transfer of drugs across the BBB with colloidal carrier systems like liposomes. Trientine (TETA) has been modified using this approach, and experiments in rats showed an up to 16-fold higher brain uptake of trientine in vectorized liposomes compared to free trientine. ${ }^{88}$ The achieved concentration would be sufficient to exhibit a therapeutic effect in WD. However, several questions remain unresolved, including the fate of liposomes and whether this approach is superior to peripherally acting chelators.

None of the available medications for WD restore copper homeostasis. Liver transplantation with a wild type of $A T P 7 B$ represents a cure for $\mathrm{WD}$, but it still results in a chronic condition because of the need for a lifelong immunosuppressive therapy in transplanted patients. However, liver transplantation is the only effective treatment in advanced liver disease with decompensated cirrhosis or fulminant liver failure because of the delay in efficacy of anticopper medications. ${ }^{89-91}$ Long-term survival of these patients is $>80 \%$, and worse prognosis is seen in patients who had fulminant course, nonelective procedure and pretransplant renal insufficiency. ${ }^{91}$

The indications for liver transplantation in hepatic disease in WD are generally established with proposed scoring systems for adults and children. ${ }^{92,93}$ However, the role of liver transplantation as a treatment of neurologic deficits remains controversial. Liver transplantation corrects the hepatic metabolic defects of WD and also affects the normalization of extrahepatic copper metabolism, including in the central nervous system. Improvement or even a complete resolution after liver transplantation has been reported in some patients with severe and progressive neurologic deficits who did not respond to conventional chelation therapies and they did not require liver transplant because of failing liver functions. ${ }^{91,94-98}$ These positive neurologic outcomes are not universal, and no improvements or further progression have been also observed. ${ }^{99,100}$ That is why liver transplantation to reverse neurologic deficits needs to be indicated with considerable caution and should be reserved for patients who progress in spite of best medical management and their neuroimaging does not show severe structural changes that would make meaningful recovery unlikely.

Cell-based therapy is another potentially useful method to restore hepatobiliary copper excretion. WD with liver failure can be treated with liver transplantation, but this requires a suitable donor and a lifelong immunosuppression with its own potential complications. Human hepatocyte transplantation is increasingly used as treatment for liverbased metabolic defects. ${ }^{101}$ Liver targeting is the most suitable approach because of nearly exclusive expression of ATP7B in hepatocytes. Transplanted hepatocytes must integrate in the liver parenchyma and reconstitute the hepatobiliary excretory apparatus in order to excrete copper into the bile canaliculus. Given the presence of disease-causing mutations, autologous cell cannot be used unless genetic defect is corrected, such as using CRISP9 technology. Mesenchymal stem cells can differentiate into hepatocytes. They are nonimmunogenic and thus, there is no need for immunosuppressive drug regimen. Mesenchymal stem cells experiments using a rat model of WD (Long-Evans Cinnamon [LEC] rats) harboring a spontaneous deletion in the $3^{\prime}$ terminal region of the $A T P 7 B$ gene demonstrated amelioration of copper 
overload. ${ }^{102}$ Recently completed human trial explored the feasibility of this approach in WD with injection of cells via the hepatic artery and peripheral vein (ClinicalTrials.gov Identifier: NCT01378182).

Delivery of a functional $A T P 7 B$ gene using gene therapy vectors could become a curative treatment for WD if sufficient transgene expression of ATP7B can be maintained in the liver for a long period of time. Previous studies demonstrated that introduction of a functional $A T P 7 B$ gene in LEC rats showed restoration of biliary copper excretion. ${ }^{103}$ However, these studies did not achieve sustained effects because of the short duration of transgene expression using the firstgeneration adenoviral vectors. Recent report using adenoassociated vector serotype 8 (AAV8) encoding the human ATP7B cDNA placed under the control of the liver-specific a1-antitrypsin promoter (AAV8-AAT-ATP7B) showed sustained benefits for 6 months in knockout mouse model of WD and correction of copper overload with normalization of biochemical abnormalities associated with WD. ${ }^{104}$ Many additional studies are needed, but this may be the dawn of gene therapy in WD.

\section{Conclusion}

These are exciting times in the WD field, and several promising novel therapies, including chelators targeting specific cell types, and cell-based and gene therapies, may revolutionize our care for WD patients. However, timely clinical diagnosis remains the main challenge. If one does not think of WD, the diagnosis is easily missed with catastrophic consequences.

\section{Disclosure}

The author reports no conflicts of interest in this work.

\section{References}

1. Bull PC, Thomas GR, Rommens JM, Forbes JR, Cox DW. The Wilson disease gene is a putative copper transporting P-type ATPase similar to the Menkes gene. Nat Genet. 1993;5(4):327-337.

2. Tanzi RE, Petrukhin K, Chernov I, et al. The Wilson disease gene is a copper transporting ATPase with homology to the Menkes disease gene. Nat Genet. 1993;5(4):344-350.

3. Brewer GJ. Wilson's Disease: A Clinician's Guide to Recognition, Diagnosis, and Management. Norwel, MA: Kluwer Academic Publishers; 2001.

4. Merle U, Schaefer M, Ferenci P, Stremmel W. Clinical presentation, diagnosis and long-term outcome of Wilson's disease: a cohort study. Gut. 2007;56(1):115-120.

5. Wilson DC, Phillips MJ, Cox DW, Roberts EA. Severe hepatic Wilson's disease in preschool-aged children. J Pediatr. 2000;137(5):719-722.

6. Ferenci P, Członkowska A, Merle U, et al. Late onset Wilson disease. Gastroenterology. 2007;132(4):1294-1298.

7. Ala A, Borjigin J, Rochwarger A, Schilsky M. Wilson disease in septuagenarian siblings: raising the bar for diagnosis. Hepatology. 2005;41(3):668-670.
8. Roberts EA, Schilsky ML; American Association for Study of Liver Diseases (AASLD). Diagnosis and treatment of Wilson's disease: an update. Hepatology. 2008;47(6):2089-2111.

9. Kalach N, Seidman EG, Morin C, et al. Acute liver failure from Wilson's disease in a five year-old child. Can J Gastroenterol. 1993;7(3): 610-612.

10. Saito T. Presenting symptoms and natural history of Wilson disease. Eur J Pediatr. 1987;146(3):261-265.

11. Oder W, Grimm G, Kollegger H, Ferenci P, Schneider B, Deecke L. Neurological and neuropsychiatric spectrum of Wilson's disease: a prospective study of 45 cases. J Neurol. 1991;238(5):281-287.

12. Machado A, Chien HF, Deguti MM, et al. Neurological manifestations in Wilson's disease: report of 119 cases. Mov Disord. 2006;21(12): 2192-2196.

13. Pellecchia MT, Criscuolo C, Longo K, Campanella G, Filla A, Barone P. Clinical presentation and treatment of Wilson's disease: a single-centre experience. Eur Neurol. 2003;50(1):48-52.

14. Prashanth LK, Taly AB, Sinha S, et al. Prognostic factors in patients presenting with severe neurological forms of Wilson's disease. $Q J$ Med. 2005;98(8):557-563.

15. Hedera P. Treatment of Wilson's disease motor complications with deep brain stimulation. Ann N Y Acad Sci. 2014;1315(5):16-23.

16. Shanmugiah A, Sinha S, Taly AB, et al. Psychiatric manifestations in Wilson's disease: a cross-sectional analysis. $J$ Neuropsychiatry Clin Neurosci. 2008;20(1):81-85.

17. Svetel M, Potrebić A, Pekmezovi T, et al. Neuropsychiatric aspects of treated Wilson's disease. Parkinsonism Relat Disord. 2009;15(10): $772-775$.

18. Walshe JM, Yealland M. Wilson's disease: the problem of delayed diagnosis. J Neurol Neurosurg Psychiatry. 1992;55(8):692-696.

19. Litwin T, Gromadzka G, Członkowska A. Gender differences in Wilson's disease. J Neurol Sci. 2012;312(1-2):31-35.

20. Azizi E, Eshel G, Aladjem M. Hypercalciuria and nephrolithiasis as a presenting sign in Wilson disease. Eur J Pediatr. 1989;148(6): $548-549$.

21. Nakada SY, Brown MR, Rabinowitz R. Wilson's disease presenting as symptomatic urolithiasis: a case report and review of the literature. J Urol. 1994;152(3):978-979.

22. Golding DN, Walshe JM. Arthropathy of Wilson's disease. Study of clinical and radiological features in 32 patients. Ann Rheum Dis. 1977;36(2):99-111.

23. Hlubocka Z, Maracek Z, Linhart A, et al. Cardiac involvement in Wilson disease. J Inherit Metab Dis. 2002;25(4):269-277.

24. Tauber J, Steinert RF. Pseudo-Kayser-Fleischer ring of the cornea associated with non-Wilsonian liver disease: a case report and literature review. Cornea. 1993;12(1):74-77.

25. Medici V, Trevisan CP, D'Inca R, et al. Diagnosis and management of Wilson's disease: results of a single center experience. J Clin Gastroenterol. 2006;40(10):936-941.

26. Demirkiran M, Jankovic J, Lewis RA, Cox DW. Neurologic presentation of Wilson disease without Kayser-Fleicher rings. Neurology. 1996;46(4):1040-1043.

27. Prashanth LK, Taly AB, Sinha S, Arunodaya GR, Swamy HS. Wilson's disease: diagnostic errors and clinical implications. J Neurol Neurosurg Psychiatry. 2004;5(6):907-909.

28. Soltanzadeh A, Soltanzadeh P, Nafissi S, Ghorbani A, Sikaroodi H, Lotfi J. Wilson's disease: a great masquerader. Eur Neurol. 2007;57(2):80-85.

29. Ferenci P, Caca K, Loudianos G, et al. Diagnosis and phenotypic classification of Wilson disease. Liver Int. 2003;23(3):139-142.

30. Cauza E, Maier-Dobersberger T, Polli C, Kaserer K, Kramer L, Ferenci P. Screening for Wilson's disease in patients with liver diseases by serum ceruloplasmin. J Hepatol. 1997;27(2):358-362.

31. Hellman ED, Gitlin JD. Ceruloplasmin metabolism and function. Annu Rev Nutr. 2002;22(7):439-458.

32. Macintyre G, Gutfreund KS, Martin WR, Camicioli R, Cox DW. Value of an enzymatic assay for the determination of serum ceruloplasmin. J Lab Clin Med. 2004;44(6):294-301. 
33. Arredondo M, Núñez H, López G, Pizarro F, Ayala M, Araya M. Influence of estrogens on copper indicators: in vivo and in vitro studies. Biol Trace Elem Res. 2010;134(3):252-264.

34. Hedera P, Peltier A, Fink JK, Wilcock S, London Z, Brewer GJ. Myelopolyneuropathy and pancytopenia due to copper deficiency and high zinc levels of unknown origin II. The denture cream is a primary source of excessive zinc. Neurotoxicology. 2009;30(6):996-999.

35. Xu X, Pin S, Gathinji M, Fuchs R, Harris ZL. Aceruloplasminemia: an inherited neurodegenerative disease with impairment of iron homeostasis. Ann N Y Acad Sci. 2004;1012:299-305.

36. Menkes JH. Menkes disease and Wilson disease: two sides of the same copper coin. Part I: Menkes disease. Eur J Paediatr Neurol. 1999;3(4):147-158.

37. Tümer Z. An overview and update of ATP7A mutations leading to Menkes disease and occipital horn syndrome. Hum Mutat. 2013;34(3): 417-429.

38. Nicastro E, Ranucci G, Vajro P, Vegnente A, Iorio R. Re-evaluation of the diagnostic criteria for Wilson disease in children with mild liver disease. Hepatology. 2010;52(6):1948-1956.

39. Rosencrantz RA, Schilsky ML. Mining for a diagnosis of Wilson's disease in children: genetics, score, and ore. Hepatology. 2010;52: 1872-1874.

40. Manolaki N, Nikolopoulou G, Daikos GL, et al. Wilson disease in children: analysis of 57 cases. J Pediatr Gastroenterol Nutr. 2009; 48(1):72-77.

41. Schilsky ML. Non-invasive testing for Wilson disease: revisiting the D-penicillamine challenge test. J Hepatol. 2007;47(2):172-173.

42. Müller T, Koppikar S, Taylor RM, et al. Re-evaluation of the penicillamine challenge test in the diagnosis of Wilson's disease in children. J Hepatol. 2007;47(2):270-276.

43. da Silva-Júnior FP, Lucato LT, Machado AA, Barbosa ER. Copper deficiency in Wilson's disease: an avoidable complication of treatment. Mov Disord. 2011;26(13):2448-2449.

44. Koppikar S, Dhawan A. Evaluation of the scoring system for the diagnosis of Wilson's disease in children. Liver Int. 2005;25(3):680-681.

45. Hermann W. Morphological and functional imaging in neurological and non-neurological Wilson's patients. Ann NY Acad Sci. 2014;1315:24-29.

46. Sinha S, Taly AB, Ravishankar S, et al. Wilson's disease: cranial MRI observations and clinical correlation. Neuroradiology. 2006; 48(9):613-621.

47. Südmeyer M, Saleh A, Wojtecki L, et al. Wilson's disease tremor is associated with magnetic resonance imaging lesions in basal ganglia structures. Mov Disord. 2006;21(12):2134-2139.

48. Hedera P, Brewer GJ, Fink JK. White matter changes in Wilson disease. Arch Neurol. 2002;59(5):866-867.

49. Weissenborn K, Bokemeyer M, Ahl B, et al. Functional imaging of the brain in patients with liver cirrhosis. Metab Brain Dis. 2004; 19(3-4):269-280.

50. Sinha S, Taly AB, Prashanth LK, Ravishankar S, Arunodaya GR, Vasudev MK. Sequential MRI changes in Wilson's disease with de-coppering therapy: a study of 50 patients. Br J Radiol. 2007;80(957):744-749.

51. Frota NA, Barbosa ER, Porto CS, et al. Cognitive impairment and magnetic resonance imaging correlations in Wilson's disease. Acta Neurol Scand. 2013;127(6):391-398.

52. Page RA, Davie CA, MacManus D, et al. Clinical correlation of brain MRI and MRS abnormalities in patients with Wilson disease. Neurology. 2004;63(4):638-643.

53. Barthel H, Hermann W, Kluge R, et al. Concordant pre- and postsynaptic deficits of dopaminergic neurotransmission in neurologic Wilson disease. AJNR Am J Neuroradiol. 2003;24(2):234-238.

54. Akpinar E, Akhan O. Liver imaging findings of Wilson's disease. Eur J Radiol. 2007;61(1):25-32.

55. Akhan O, Akpinar E, Karcaaltincaba M, et al. Imaging findings of liver involvement of Wilson's disease. Eur J Radiol. 2009;69(1):147-155.

56. Sini M, Sorbello O, Civolani A, Liggi M, Demelia L. Non-invasive assessment of hepatic fibrosis in a series of patients with Wilson's Disease. Dig Liver Dis. 2012;44(6):487-491.
57. Lutsenko S, Barnes NL, Bartee MY, Dmitriev OY. Function and regulation of human copper-transporting ATPases. Physiol Rev. 2007;87(3):1011-1046.

58. Olivarez L, Caggana M, Pass KA, Ferguson P, Brewer GJ. Estimate of the frequency of Wilson's disease in the US Caucasian population: a mutation analysis approach. Ann Hum Genet. 2001; 65(5):459-463.

59. Olsson C, Waldenstrom E, Westermark K, Landegre U, Syvanen AC. Determination of the frequencies of ten allelic variants of the Wilson disease gene (ATP7B), in pooled DNA samples. Eur J Hum Genet. 2000;8(12):933-938.

60. Hahn SH, Lee SY, Jang YJ, et al. Pilot study of mass screening for Wilson's disease in Korea. Mol Genet Metab. 2002;76(2): $133-136$.

61. Loudianos G, Dessi V, Lovicu M, et al. Molecular characterization of Wilson disease in the Sardinian population - evidence of a founder effect. Hum Mutat. 1999;14(4):294-303.

62. Rodriguez-Castro KI, Hevia-Urrutia FJ, Sturniolo GC. Wilson's disease: a review of what we have learned. World J Hepatol. 2015; 7(29):2859-2870.

63. Coffey AJ, Durkie M, Hague S, et al. A genetic study of Wilson's disease in the United Kingdom. Brain. 2013;136(5):1476-1487.

64. Thomas GR, Forbes JR, Roberts EA, Walshe JM, Cox DW. The Wilson disease gene: spectrum of mutations and their consequences. Nat Genet. 1995;9(2):210-217.

65. Ferenci P. Phenotype-genotype correlations in patients with Wilson's disease. Ann N Y Acad Sci. 2014;1315(5):1-5.

66. Okada T, Shiono Y, Kaneko Y, et al. High prevalence of fulminant hepatic failure among patients with mutant alleles for truncation of ATP7B in Wilson's disease. Scand J Gastroenterol. 2010; 45(10):1232-1237.

67. Nicastro E, Loudianos G, Zancan L, et al. Genotype-phenotype correlation in Italian children with Wilson's disease. J Hepatol. 2009;50(3):555-561.

68. Houwen RH, Juyn J, Hoogenraad TU, Ploos van Amstel JK, Berger R. $\mathrm{H} 714 \mathrm{Q}$ mutation in Wilson disease is associated with late, neurological presentation. J Med Genet. 1995;32(6):480-482.

69. Caca K, Ferenci P, Kühn HJ, et al. High prevalence of the H1069Q mutation in East German patients with Wilson disease: rapid detection of mutations by limited sequencing and phenotype-genotype analysis. J Hepatol. 2001;35(5):575-581.

70. Noureen N, Rana MT. Neurological Wilson disease in children: a three tears experience from Multan. J Pak Med Assoc. 2011;61(8): 734-738.

71. Usta J, Wehbeh A, Rida K, et al. Phenotype-genotype correlation in Wilson disease in a large Lebanese family: association of c.2299insC with hepatic and p.Ala1003Thr with neurologic phenotype. PLoS One. 2014;9(11):e109727.

72. Al Fadda M, Al Quaiz M, Ashgar H, et al. Wilson disease in 71 patients followed for over two decades in a tertiary center in Saudi Arabia: a retrospective study. Ann Saudi Med. 2012;32(6):623-629.

73. Chabik G, Litwin T, Członkowska A. Concordance rates of Wilson's disease phenotype among siblings. $J$ Inherit Metab Dis. 2014;37(1):131-135.

74. Brewer GJ, Johnson VD, Dick RD, Hedera P, Fink JK, Kluin KJ. Treatment of Wilson's disease with zinc. XVII: treatment during pregnancy. Hepatology. 2000;31(2):364-370.

75. Walshe JM. Pregnancy in Wilson's disease. Q J Med. 1977;46(181): $73-83$.

76. Walshe JM. The management of pregnancy in Wilson's disease treated with trientine. Q J Med. 1986;58(225):81-87.

77. Devesa R, Alvarez A, de las Heras G, Ramon de Miguel J. Wilson's disease treated with trientine during pregnancy. J Pediatr Gastroenterol Nutr. 1995;20(1):102-103.

78. Brewer GJ, Terry CA, Aisen AM, Hill GM. Worsening of neurologic syndrome in patients with Wilson's disease with initial penicillamine therapy. Arch Neurol. 1987;44(5):490-493. 
79. Brewer GJ, Askari F, Lorincz MT, et al. Treatment of Wilson disease with ammonium tetrathiomolybdate: IV. Comparison of tetrathiomolybdate and trientine in a double-blind study of treatment of the neurologic presentation of Wilson disease. Arch Neurol. 2006;63(4):521-527.

80. Brewer GJ, Dick RD, Yuzbasiyan-Gurkin V, Tankanow R, Young AB, Kluin KJ. Initial therapy of patients with Wilson's disease with tetrathiomolybdate. Arch Neurol. 1991;48(1):42-47.

81. Brewer GJ, Dick RD, Johnson V, et al. Treatment of Wilson's disease with ammonium tetrathiomolybdate. I. Initial therapy in 17 neurologically affected patients. Arch Neurol. 1994;51(6):545-554.

82. Brewer GJ, Johnson V, Dick RD, Kluin KJ, Fink JK, Brunberg JA. Treatment of Wilson disease with ammonium tetrathiomolybdate. II. Initial therapy in 33 neurologically affected patients and follow-up with zinc therapy. Arch Neurol. 1996;53(10):1017-1025.

83. Brewer GJ, Hedera P, Kluin KJ, et al. Treatment of Wilson disease with ammonium tetrathiomolybdate: III. Initial therapy in a total of 55 neurologically affected patients and follow-up with zinc therapy. Arch Neurol. 2003;60(3):379-385.

84. Brewer GJ, Askari F, Dick RB, et al. Treatment of Wilson's disease with tetrathiomolybdate: V. Control of free copper by tetrathiomolybdate and a comparison with trientine. Transl Res. 2009;154(2):70-77.

85. Jullien AS, Gateau C, Lebrun C, Kieffer I, Testemale D, Delangle P. D-Penicillamine tripodal derivatives as efficient copper(I) chelators. Inorg Chem. 2014;53(10):5229-5239.

86. Gateau C, Delangle P. Design of intrahepatocyte copper(I) chelators as drug candidates for Wilson's disease. Ann N Y Acad Sci. 2014;1315(5):30-36.

87. Stuerenburg HJ. CSF copper concentrations, blood-brain barrier function, and ceruloplasmin synthesis during the treatment of Wilson's disease. J Neural Transm. 2000;107(3):321-329.

88. Tremmel R, Uhl P, Helm F, et al. Delivery of copper-chelating trientine (TETA) to the central nervous system by surface modified liposomes. Int J Pharm. 2016;512(1):87-95.

89. Schilsky ML, Scheinberg IH, Sternlieb I. Liver transplantation for Wilson's disease: indications and outcome. Hepatology. 1994;19(3):583-587.

90. Eghtesad B, Nezakatgoo N, Geraci LC, et al. Liver transplantation for Wilson's disease: a single-center experience. Liver Transpl Surg. 1999;5(6):467-474.

91. Guillaud O, Dumortier J, Sobesky R, et al. Long term results of liver transplantation for Wilson's disease: experience in France. J Hepatol. 2014;60(3):579-589.
92. Dhawan A, Taylor RM, Cheeseman P, De Silva P, Katsiyiannakis L, Mieli-Vergani G. Wilson's disease in children: 37-year experience and revised King's score for liver transplantation. Liver Transpl. 2005; 11(4):441-448.

93. Petrasek J, Jirsa M, Sperl J, et al. Revised King's College score for liver transplantation in adult patients with Wilson's disease. Liver Transpl. 2007;13(1):55-61.

94. Bax RT, Hassler A, Luck W, et al. Cerebral manifestation of Wilson's disease successfully treated with liver transplantation. Neurology. 1998;51(3):863-865.

95. Lui CC, Chen CL, Cheng YF, Lee TY. Recovery of neurological deficits in a case of Wilson's disease after liver transplantation. Transplant Proc. 1998;30(7):3324-3325.

96. Stracciari A, Tempestini A, Borghi A, Guarino M. Effect of liver transplantation on neurological manifestations in Wilson disease. Arch Neurol. 2000;57(3):384-386.

97. Mocchegiani F, Gemini S, Vincenzi P, et al. Liver transplantation in neurological Wilson's disease: is there indication? A case report. Transplant Proc. 2014;46(7):2360-2364.

98. Laurencin C, Brunet AS, Dumortier J, et al. Liver transplantation in Wilson's disease with neurological impairment: evaluation in 4 patients. Eur Neurol. 2016;77(1-2):5-15.

99. Guarino M, Stracciari A, D’Alessandro R, Pazzaglia P. No neurological improvement after liver transplantation for Wilson's disease. Acta Neurol Scand. 1995;92(5):405-408.

100. Kassam N, Witt N, Kneteman N, Bain VG. Liver transplantation for neuropsychiatric Wilson disease. Can J Gastroenterol. 1998;12(1): 65-68.

101. Filippi C, Dhawan A. Current status of human hepatocyte transplantation and its potential for Wilson's disease. Ann NY Acad Sci. 2014;1315(5):50-55.

102. Chen S, Shao C, Dong T, et al. Transplantation of ATP7B-transduced bone marrow mesenchymal stem cells decreases copper overload in rats. PLoS One. 2014;9(11):e111425.

103. Ha-Hao D, Merle U, Hofmann C, et al. Chances and shortcomings of adenovirus-mediated ATP7B gene transfer in Wilson disease: proof of principle demonstrated in a pilot study with LEC rats. $Z$ Gastroenterol. 2002;40(4):209-216.

104. Murillo O, Luqui DM, Gazquez C, et al. Long-term metabolic correction of Wilson's disease in a murine model by gene therapy. J Hepatol. 2016;64(2):419-426.
The Application of Clinical Genetics

\section{Publish your work in this journal}

The Application of Clinical Genetics is an international, peer-reviewed open access journal that welcomes laboratory and clinical findings in the field of human genetics. Specific topics include: Population genetics; Functional genetics; Natural history of genetic disease; Management of genetic disease; Mechanisms of genetic disease; Counselling and ethical

\section{Dovepress}

issues; Animal models; Pharmacogenetics; Prenatal diagnosis; Dysmorphology. The manuscript management system is completely online and includes a very quick and fair peer-review system, which is all easy to use. Visit http://www.dovepress.com/testimonials.php to read real quotes from published authors. 